\title{
Novel Pharmacological Treatment of Post-prandial Hyperinsulinemic Hypoglycemia
}

\author{
Emily Cooper* \\ Diabesity Institute, USA
}

Submission: January 15, 2019; Published: February 22, 2019

*Corresponding author: Emily Cooper, Diabesity Institute, 2320 W. Commodore Way, Suite 100, Seattle, WA 98199, USA

\begin{abstract}
Background: Post Roux-en-Y gastric bypass (RYGB) hyperinsulinemia hypoglycemia (HH) is presumably related to enhanced glucagon-like peptide-1 (GLP-1) response with partial pancreatectomy as recommended treatment. A new approach successfully uses short-acting GLP-1 mimetics, challenging assumptions about post-RYGB HH etiology and standard treatment.

Case Description: A 61-year-old woman with lifelong obesity leading to RYGB at 304 pounds followed by 10 years of fatigue and weight fluctuation initially lost 100 pounds, regained 80 pounds, then lost 60 pounds with aggressive diet and exercise. Ten years post-RYGB, she presented with extreme fatigue and weight regain. Mixed-meal tolerance testing revealed post prandial HH: 30-minute post-prandial insulin response of $550.8 \mathrm{mU} / \mathrm{mL} ; 60$ - and 90 -minute post-prandial glucose of 48 and $56 \mathrm{mg} / \mathrm{dL}$, respectively; weight of 219 pounds, BMI 36.4 . Initial treatment included pre-meal acarbose $50 \mathrm{mg}$ and exenatide $5 \mu \mathrm{g}$ three times/day, balanced meals and snacks, and no prescribed exercise. Insulin and glucose levels improved immediately accompanied by weight loss. Acarbose and exenatide doses were adjusted until the insulin response stabilized. Zonisamide $(50 \mathrm{mg})$ and naltrexone $(25 \mathrm{mg})$ twice/day were also prescribed after detecting elevated agouti-related peptide. By 12 months, the patient's weight was 157 pounds (28\% weight loss) and BMI 26.1. By 18 months, her weight was 140 pounds (36\% weight loss) and BMI 23.3.
\end{abstract}

Conclusions: This report is the first to describe effective use of short-acting exenatide combined with an alpha-glucoside inhibitor to regulate post-RYGB post-prandial $\mathrm{HH}$ and facilitate concomitant weight loss, and it challenges previous assumptions regarding post-prandial $\mathrm{HH}$ etiology and invasive treatment with partial pancreatectomy.

Keywords: Post-prandial hyperinsulinemic hypoglycemia; GLP-1 mimetics; nesidioblastosis; pharmacological agents

Abbreviations: AgRP: agouti-related protein; GIP: glucose-dependent insulinotropic polypeptide; GLP-1: glucagon-like peptide-1; HH: hyperinsulinemia hypoglycemia; LDL: low-density lipoprotein; MSH: melanocyte-stimulating hormone; PBSH: post-bariatric surgery hypoglycemia; RMR: resting metabolic rate; RYGB: Roux-en-Y gastric bypass

\section{Introduction}

Bariatric surgery is described as the most effective intervention for weight loss with Roux-en-Y gastric bypass (RYGB) considered the gold standard operation; however, partial weight regain is not uncommon [1]. A subset of patients who have undergone RYGB experience post-prandial hyperinsulinemia hypoglycemia $(\mathrm{HH})$, which presumably is related to enhanced post-surgical glucagon-like peptide-1 (GLP-1) response [1,2]. The etiology of the partial weight regain seen in RYGB is unclear, although, speculation is that gut and adipocyte hormones and neuropeptides play a role in regulating insulin and glucose levels provoking weight gain and obesity [3]. For those patients with post-prandial $\mathrm{HH}$ that does not respond to nutritional changes, other treatments including gastric bypass reversal or partial pancreatectomy have been used. Additionally, pharmacological treatments including calcium channel blockade, ocreotide, diazoxide, and alpha glucosidase inhibition, have been effective at improving insulin and glucose regulation in patients with non-insulinoma post-prandial $\mathrm{HH}[4]$.

This case study describes the novel pharmacological treatment of a female patient with obesity who experienced extreme fatigue and fluctuations in weight for 10 years postRYGB surgery.

\section{Case Report}

A 61-year-old Caucasian female experienced failed weight regulation during most of her life despite regularly dieting and exercising. Family history includes a brother with adult-onset obesity; paternal grandfather with severe obesity; and paternal 


\section{Current Research in Diabetes \& Obesity Journal}

aunts and uncles who were obese. Other family characteristics include hypoglycemia with neuroglycopenic symptoms (father and nephew) and alcoholism (father and brother).

The patient's history includes normal weight at birth and as a child; however, in middle school, her weight increased, which was when the patient started her first diet. Over time, dieting included commercial weight loss programs and diet meals or calorie counting. In 2005, when 52 years old, and before undergoing bariatric surgery, the patient weighed 304 pounds. In 2007, two years after RYGB, she weighed approximately 204 pounds and remained at or near that weight until October 2014, prior to retirement from work, when her weight increased to 284 pounds. After retirement, the patient participated in an aggressive diet and exercise routine and lost approximately 70 pounds, but also experienced extreme fatigue.

On April 6, 2015, the patient presented to the clinic with extreme fatigue, palpitations, sweating, and migraine. She weighed 211 pounds, her height was 5 feet 5 inches, and BMI was 35.1. On June 15, 2015, the patient weighed 219 pounds; blood pressure was 126/87; and BMI was 36.4. Her levels of insulin and glucose were normal $(7.2 \mathrm{mU} / \mathrm{mL}$ and $97 \mathrm{mg} / \mathrm{dL}$, respectively) while levels of the following were high: HbA1c (5.9\%); total cholesterol (248 mg/dL), and low-density lipoprotein (LDL) (125 mg/dL). Leptin was normal, melanocyte-stimulating hormone (MSH) was elevated (tested at LabCorp; Seattle, WA), and post-prandial GLP 1 levels (tested at Interscience Institute; Inglewood, CA) failed to rise following consumption of a mixed meal. Mixed-meal tolerance testing showed a markedly elevated insulin response, particularly at the 30 -minute postprandial time point $(550.8 \mathrm{mU} / \mathrm{mL})$, and low glucose levels at the 60 - and 90 -minute post-prandial time points $(48 \mathrm{mg} / \mathrm{dL}$ and $56 \mathrm{mg} / \mathrm{dL}$, respectively) (Table 1 ). Genetic testing for congenital hyperinsulinism was negative (tested at Athena Diagnostics; Marlborough, MA).

Table 1: Post-prandial Insulin and Glucose Levels.

\begin{tabular}{|c|c|c|c|c|c|c|c|c|c|c|c|c|c|c|c|}
\hline \multirow{3}{*}{$\begin{array}{l}\text { Treatment } \\
\text { Date }\end{array}$} & \multicolumn{5}{|c|}{ Insulin $(\mathrm{mU} / \mathrm{mL})$} & \multicolumn{5}{|c|}{ Glucose $(\mathrm{mg} / \mathrm{dL})$} & \multicolumn{5}{|c|}{ Insulin/Glucose (\%) } \\
\hline & \multicolumn{5}{|c|}{ Minutes After Meal } & \multicolumn{5}{|c|}{ Minutes After Meal } & \multicolumn{5}{|c|}{ Minutes After Meal } \\
\hline & 0 & 30 & 60 & 90 & 120 & 0 & 30 & 60 & 90 & 120 & 0 & 30 & 60 & 90 & 120 \\
\hline June $2015^{\mathrm{a}}$ & 7.2 & 550.8 & 79.3 & 20.6 & -- & 97 & 122 & 48 & 56 & -- & 7.4 & 451.5 & 165.2 & 36.8 & -- \\
\hline June $2016^{\mathrm{b}}$ & -- & 121.7 & 35 & 10.7 & -- & -- & 99 & 62 & 74 & -- & -- & 122.9 & 56.5 & 14.5 & -- \\
\hline Aug $2016^{c}$ & -- & 33.5 & 28.6 & 12.8 & 6.9 & -- & 112 & 116 & 103 & 98 & -- & 29.9 & 24.7 & 12.4 & 7 \\
\hline Dec $2016^{c}$ & 7.4 & 25.1 & 10.4 & 7.8 & -- & 87 & 85 & 76 & 81 & -- & 8.5 & 29.5 & 13.7 & 9.6 & -- \\
\hline
\end{tabular}

a: Pre-treatment; b: Treatment initiated with acarbose $(50 \mathrm{mg})$ and exenatide $(5 \mu \mathrm{g})$; c: Treatment with exenatide $(15 \mu \mathrm{g})$

Pharmacological treatment was initiated on June 23, 2015 with the primary goal of controlling post-prandial HH. Treatment included pre-meal acarbose $50 \mathrm{mg}$ and short-acting exenatide $5 \mu \mathrm{g}$ three times/day; these doses were adjusted during treatment to stabilize the post-prandial insulin response. Zonisamide (50 $\mathrm{mg}$ ) and naltrexone (25 $\mathrm{mg}$ ) twice/day were also added to the treatment regimen in October 2015 to counteract potential effects of elevated circulating agouti-related protein (AgRP) (88 pg/mL). By August 2016, the 30-minute post-prandial insulin level stabilized $(33.5 \mathrm{mU} / \mathrm{mL}), 14$ months after pharmacological treatment was initiated. Additionally, by December 2016, the post-prandial 60- and 90- minute glucose levels stabilized $(76 \mathrm{mg} / \mathrm{dL}$ and $81 \mathrm{mg} / \mathrm{dL}$, respectively), 18 months after treatment started.

The patient's weight was recorded at periodic visits to the clinic during treatment with a steady decline in weight. Four months after initiation of treatment (October 2015), the patient's weight dropped from the pre-treatment weight of 219 pounds to 201 pounds; it was reduced further to 184 pounds by the end of that year (December 2015), and then to 157 pounds at 12 months from initiation of treatment (June 2016) for 28\% loss in weight (Figure 1). By 18 months of treatment (December 2016), the patient's weight was 140 pounds ( $36 \%$ weight loss, BMI
23.3). The next weight taken during this observation period was one month later (January 2017) and it was 136 pounds (38\% weight loss, BMI 22.6).

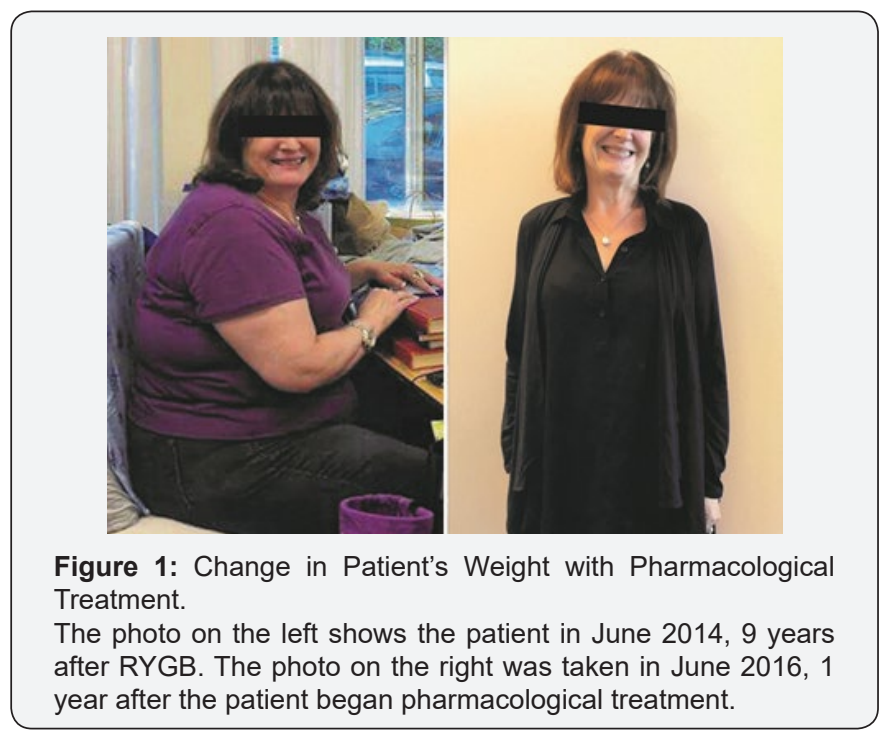

Blood pressure was well controlled and approximately 120/80 mmHg during this 18-month period of treatment and observation. During this same period other measurements also were under control such as $\mathrm{HbA1c}$ with readings as low as $5.1 \%$ and as 


\section{Current Research in Diabetes \& Obesity Journal}

high as 5.7\%; total cholesterol, $207 \mathrm{mg} / \mathrm{dL}$ and $250 \mathrm{mg} / \mathrm{dL}$; and low-density lipoprotein (LDL), $86 \mathrm{mg} / \mathrm{dL}$ and $127 \mathrm{mg} / \mathrm{dL}$.

After a calorie-restriction approach to weight loss, a patient's resting metabolic rate (RMR) is usually suppressed. In this case study, after weight loss using a pharmacological approach, the patient's predicted RMR was 1255 calories/day whereas her actual measured RMR was normal at 1675 calories/day (Cosmed CPET).

During treatment, the patient consumed food from all food groups throughout the day without counting calories, and she participated in limited exercise until glucose levels were stable, after which the patient was advised to avoid prolonged exercise sessions or high-intensity exercise.

This case demonstrates that pharmacological treatment can effectively control post-prandial $\mathrm{HH}$ by stabilizing insulin and glucose levels in a patient with extreme fatigue related to post-prandial $\mathrm{HH}$ and weight regain after RYGB surgery. The mechanisms of treatment efficacy have yet to be elucidated; however, they may include improvement in neuro-regulation of the immediate post-prandial response via central metabolic pathway activation or peripheral hormonal effects.

\section{Discussion}

Obesity is a complex disease commonly managed with diet and exercise, and when that fails, bariatric surgery and pharmacological agents. This case identifies components of metabolic dysfunction in a patient who underwent RYGB but struggled with extreme fatigue and cycles of weight regain for 10 years after surgery.

Understanding the underlying components of metabolic dysfunction allows for a better targeted approach to pharmacological treatment to partially reverse the dysfunction. With attention to post-prandial insulin and glucose response and comprehensive laboratory testing of key metabolic hormones and neuropeptides, it is possible to achieve long-term control of post-prandial $\mathrm{HH}$ and obesity. In this case report, improvements occurred immediately with treatments to stabilize the postprandial insulin response resulting in fewer and less severe neuroglycopenic symptoms, less fatigue, and consistent weight loss. Multiple adjustments were made to the doses before normalization of insulin and glucose responses, which remained in control at the timepoint of 18 months after initiation of treatment.

Lee et al. found that post-bariatric surgery hypoglycemia (PBSH) symptoms were common, affecting up to $34 \%$ of the overall population [5]. This figure was considered an underestimate as PBSH is difficult to recognize and usually it is not monitored in this patient population. Additionally, preoperative hypoglycemia symptoms were the strongest indicator of elevated risk of postbariatric surgery hypoglycemia, regardless of preoperative diabetes status. Nannipieri et al. [6] determined that lower pre-surgery plasma glucose concentrations, higher insulin sensitivity, and better $\beta$-cell glucose sensitivity were significant predictors of post-prandial hypoglycemia after RYGB.

To determine the preferred treatment of post-RYBG hypoglycemia, Lee et al. [7] tested the effects of RYGB reversal and found that after a mixed-meal test, hyperinsulinemia hypoglycemia was not lessened and that the increase in glucose-dependent insulinotropic polypeptide (GIP) levels and the simultaneous decrease in GLP-1 levels indicated a role of GIP in ongoing hyperinsulinemia hypoglycemia.

Partial or full pancreatectomy to treat hyperinsulinemia hypoglycemia after RYGB was successful in resolving symptoms among $67 \%$ of patients in one analysis [8]. However, because of the short follow-up period in that analysis, the effectiveness of pancreatectomy remains unclear. Additionally, the associated morbidity of this procedure and the availability of other treatments, such as pharmacological agents, makes pancreatectomy less favorable.

Published reports on the pharmacological approach to managing hyperinsulinemia hypoglycemia indicate that a GLP1 receptor antagonist (exendin-[9-39]) corrected post-prandial hypoglycemia in patients who underwent gastric bypass surgery, suggesting that increased GLP-1 activity contributed to the hypoglycemia [9]. Mordes et al. [4] reported the efficacious management of hyperinsulinemic hypoglycemia post-RYGB with medical therapy (calcium-channel blocker, alpha-glucosidase inhibitor, or combination of both), and Abrahammson and others observed a protective effect of long-acting GLP-1 analogs on post-prandial hypoglycemic episodes in patients one to four years after gastric bypass surgery [10].

These publications and many others found in the literature describe different treatments that center around GLP-1. The case reported here contributes to that body of information by describing the successful, but less known use of short-acting GLP-1 analogs for the treatment of hyperinsulinemia hypoglycemia. The author has taken this approach to treatment based on her clinical experience in which short-acting GLP-1 analogs have been more effective than long-acting analogs at controlling post-prandial HH. This observed difference may be attributed to the rapid onset of action at meal time from short-acting GLP-1 which simulates normal GLP 1 response. This activity is in contrast to the longer-acting GLP-1 medications which do not provide the same biological simulation of the body's metabolism.

Hypoglycemia increases hypothalamic AgRP with an inhibitory effect on MC4 receptor activation, contributing to hyperinsulinemia and potential subsequent hypoglycemia. This cycle increases susceptibility to weight regain, hyperinsulinia, and hypoglycemia due to chronic disruption of MC4 receptor activation. The optimal treatment to break this cycle remains to be determined. 


\section{Conclusion}

Pharmacological treatment is one of several effective tools available to physicians when caring for patients with metabolic dysfunction leading to imbalances in insulin and glucose and chronic problems in managing weight. Short-acting GLP-1 mimetics with alpha glucosidase inhibition can be effective clinically despite the prior assumption that GLP-1 excess is potentially an etiology for this condition. This case report explains how the use of GLP-1 mimetics with alpha glucosidase inhibitors is one option in a group of pharmacological agents with the potential for managing post-prandial $\mathrm{HH}$.

\section{Acknowledgment}

Christine Kuepfer assisted with the preparation of this case report.

\section{Conflict of Interest}

A private grant from Gregg Mamikunian supported the writing of this case report. The author has no conflict of interest to disclose.

\section{References}

1. Moroni R, Zappa MA, Fantola G, Carbonelli MG, Micanti F (2017) The problem of weight regain, In: Angrisani L, (Edt.), Bariatric and metabolic surgery, updates in surgery (Chapter 13). Springer-Verlag Italia, pp. 978-988.

2. Service GJ, Thompson GB, Service FJ, Andrews JC, Collazo-Clavell ML, et al. (2005) Hyperinsulinemic hypoglycemia with nesidioblastosis after gastric-bypass surgery. N Engl J Med 353(3): 249-254.
3. Lean MEJ, Malkova D (2016) Altered gut and adipose tissue hormones in overweight and obese individuals: cause or consequence? Int J Obes 40(4): 622-632.

4. Mordes JP, Alonso LC (2015) Evaluation, medical therapy, and course of adult persistent hyperinsulinemic hypoglycemia after Roux-En-Y gastric bypass surgery: a case series. Endocr Pract 21(3): 237-246.

5. Lee CJ, Clark JM, Schweitzer M, Magnuson T, Steele K, et al. (2015) Prevalence of hypoglycemic symptoms after Roux-en-Y gastric bypass and vertical sleeve gastrectomy and associated risk factors. Obesity 23(5): 1079-1084.

6. Nannipieri M, Belligoli A, Guarino D, Busetto L, Moriconi D, et al. (2016) Risk factors for spontaneously self-reported postprandial hypoglycemia after bariatric surgery. J Clin Endocrinol Metab 101(11): 3600-3607.

7. Lee CJ, Brown T, Magnuson TH, Egan JM, Carlson O, et al. (2013) Hormonal response to a mixed-meal challenge after reversal of gastric bypass for hypoglycemia. Clin Endocrinol Metab 98(7): E1208-E1212.

8. Mala T (2014) Postprandial hyperinsulinemic hypoglycemia after gastric bypass surgical treatment. Surg Obes Relat Dis 10(6): 12201225.

9. Salehi M, Gastaldelli A, D’Alessio DA (2014) Blockade of glucagon-like peptide 1 receptor corrects postprandial hypoglycemia after gastric bypass gastroenterology. Gastroenterology 146(3): 669-680.

10. Abrahamsson N, Engström BE, Sundbom M, Karlsson FA (2013) GLP1 analogs as treatment of postprandial hypoglycemia following gastric bypass surgery: a potential new indication? Eur J Endocrinol 169(6): 885-889.

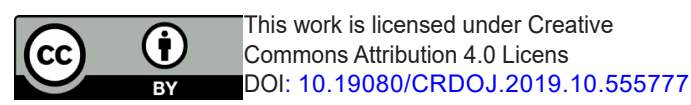

